\title{
LA ELABORACIÓN DE LO DECORATIVO: UN ACERCAMIENTO COMPARATIVO HACIA LOS ASPECTOS TÉCNICOS DE LA ALFARERÍA INCISA DE LOS RÍOS PARANÁ Y URUGUAY
}

\author{
Flavia V. Ottalagano ${ }^{1}$ y Juan C. Castro ${ }^{2}$ \\ ${ }^{1}$ CONICET/Escuela de Antropología, FHyA, UNR. flaviaott7@gmail.com, \\ ${ }^{2}$ CONICET/Dpto. Científico de Arqueología, FCNyM, UNLP/Museo de Cs. Nat. y Antrop."Prof. A. Serrano". \\ castro_museoserrano@yahoo.com.ar
}

Presentado el: 04/11/2011 - Aceptado 05/12/2011

\section{Introducción}

La incisión es una técnica decorativa que consiste en el trazado de diseños cuando la pasta se encuentra en estado plástico o con la consistencia del cuero. La incisión de punto y de surco rítmico constituyen dos variaciones de esta técnica; caracterizadas, en el primer caso, por la realización de puntos o marcas de manera individual con la ayuda de un instrumento punzante de diversas formas, y en el segundo caso, mediante la variación regular de la presión ejercida por el instrumento en movimiento (Convención Nacional de Antropología 1966). Tradicionalmente, los estudios sobre decoración cerámica de las poblaciones prehispánicas asentadas sobre las Tierras Bajas del río Paraná y del río Uruguay, se enfocaron mayormente en los aspectos formales y estilísticos (e.g. Cione et al. 1977; Badano 1957; Serrano 1946, 1950, 1954), relegando lo concerniente a su elaboración técnica. Sólo recientemente se han desarrollado avances es este camino, incorporando los aportes de la experimentación (Ceruti 2001; Frère et al. 2004; González et al. 2007, entre otros).

Este trabajo presenta los resultados preliminares de los estudios comparativos realizados entre la alfarería incisa proveniente de la cuenca media e inferior del río Paraná y de la cuenca inferior del río Uruguay (provincia de Entre Ríos), asociada a grupos cazadoresrecolectores-pescadores del último tramo del Holoceno tardío ${ }^{1}$. En particular, se analizan las características de estas improntas incisas (e.g. morfología, dimensiones, profundidad), evaluándoselas en relación a aquellas realizadas experimentalmente en el marco de trabajos previos (Ottalagano 2010). La intención es poder señalar el posible instrumental utilizado, así como los gestos técnicos implicados en la realización de la decoración, comparando diferencias y similitudes a nivel de sectores.

\section{Materiales y métodos}

La muestra analizada se compone de 1491 fragmentos de vasijas y recipientes enteros, decorados mediante técnicas de incisión de surco rítmico y/o incisión de punto. Proviene de 14 sitios arqueológicos distribuidos en los tres sectores mencionados, y son (figura 1): 


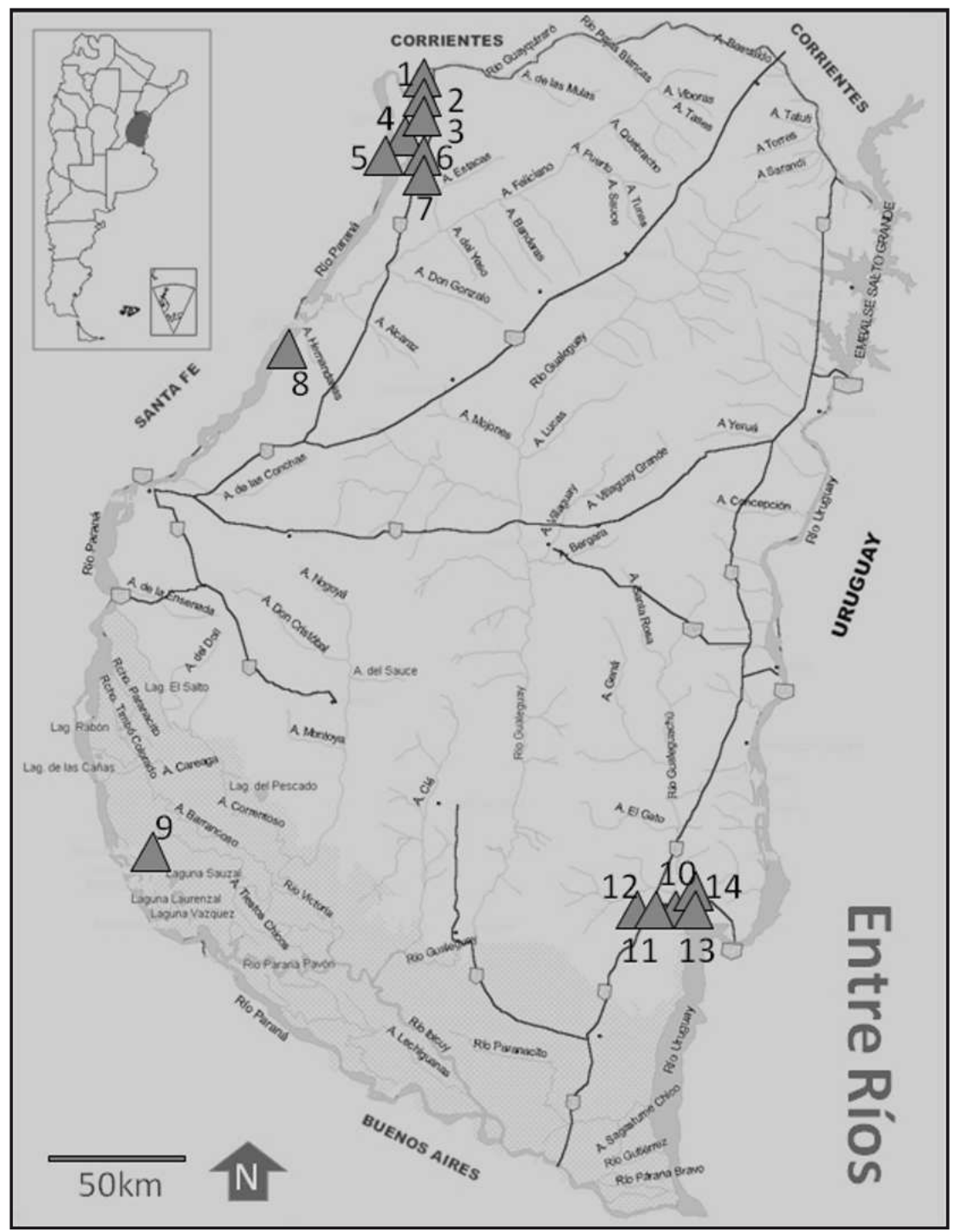

Figura 1. Localización de los sitios abordados: 1) PC1; 2) PC2; 3) LM1;4) EL1; 5) EL5; 6) A1; 7) A4; 8) LP2; 9) CPA; 10) CDB3; 11) CL2; 12) CY; 13) EP1; 14) EP2.

a) Puerto Cuartel 1 y 2 (PC1 y PC2), A . Las Mulas 1 (LM1), A ${ }^{\circ}$. El Largo 1 y 5 (EL1 y EL5), $\mathrm{A}^{\circ}$. Arenal 1 y 4 (A1 y A4) y La Palmera 2 (LP2), ubicados en el Paraná medio, en los Dptos. Paraná y La Paz (Ceruti 2003); b) Cerro Puesto Acosta (CPA), en el Delta superior del Paraná, en el Dpto. Victoria (Ottalagano et al. 2010); c) Cerro de Boari 3 (CDB3), Cerro Lorenzo 2 (CL2), Cerro Yaguar-í (CY) y El Pinar 1 y 2 (EP1 y EP2), localizados en Uruguay inferior, en el Dpto. Gualeguaychú (Castro 2011). La muestra abordada incluye también la colección Casella, procedente del Dpto. Gualeguaychú, así como parte de los materiales de la colección Serrano, del Dpto. La Paz (ver Serrano 1946); materiales que se encuentran actualmente en el Museo de Ciencias Naturales y Antropología "Profesor A. Serrano" de la ciudad de Paraná. Tanto los sitios localizados sobre el río Uruguay, como el del Delta superior del Paraná, se 
encuentran en proceso de excavación; por lo que se espera que la muestra, particularmente de estos sectores, pueda ser ampliada a futuro.

El análisis tuvo en cuenta dos grandes grupos de variables destinadas a recuperar información relacionada con los aspectos tecnológicos de la decoración: por un lado, respecto a los posibles elementos utilizados como instrumental, y por el otro, respecto a los gestos técnicos implementados por los alfareros para elaborar lo decorativo. En tal sentido, se evaluó la morfología general y dimensiones de las improntas arqueológicas; considerándose también otras variables, como el estado de secado de la arcilla al momento de la decoración, la profundidad de las huellas incisas, la orientación y el ángulo de inclinación con el que potencialmente pudo haber sido utilizado el instrumento. Como marco de referencia para esto, se tomaron en cuenta las observaciones realizadas en un trabajo experimental previo (Ottalagano 2010), en el que se efectuaron decoraciones con diversos elementos, potencialmente disponibles para los alfareros: bordes cerámicos (labios rectos y convexos); madera y vegetal: ramas, espinas y frutos de espinillo (Acacia caven) y tallos de paja brava (Panicum prionitis); plumas de Psitácidas y Falcónidas; fragmentos de valvas de Diplodon; huesos planos y huesos largos (fracturados y completos) de mamíferos medianos y pequeños; espina dorsal de Doradidae; e incisivos completos de coypo. Los mismos, no fueron sometidos a modificaciones específicas para formatizarlos, ni fueron enmangados para su uso. Por otra parte, en este trabajo se adicionó, además, algunos datos con respecto a la decoración experimental con puntas óseas, no contempladas en el trabajo anterior.

Las experiencias se realizaron sobre muestras de arcilla con diferentes niveles de secado (plástica, dureza de cuero). Los tiestos experimentales se elaboraron con arcilla extraída de la cuenca del río Paraná, específicamente de las barrancas próximas al sitio CPA. Los distintos elementos mencionados, se utilizaron variando aspectos vinculados con la orientación de los mismos y con su inclinación. Es decir, se procedió a efectuar improntas orientando los instrumentos perpendicularmente y horizontalmente a la superficie arcillosa. Asimismo, se

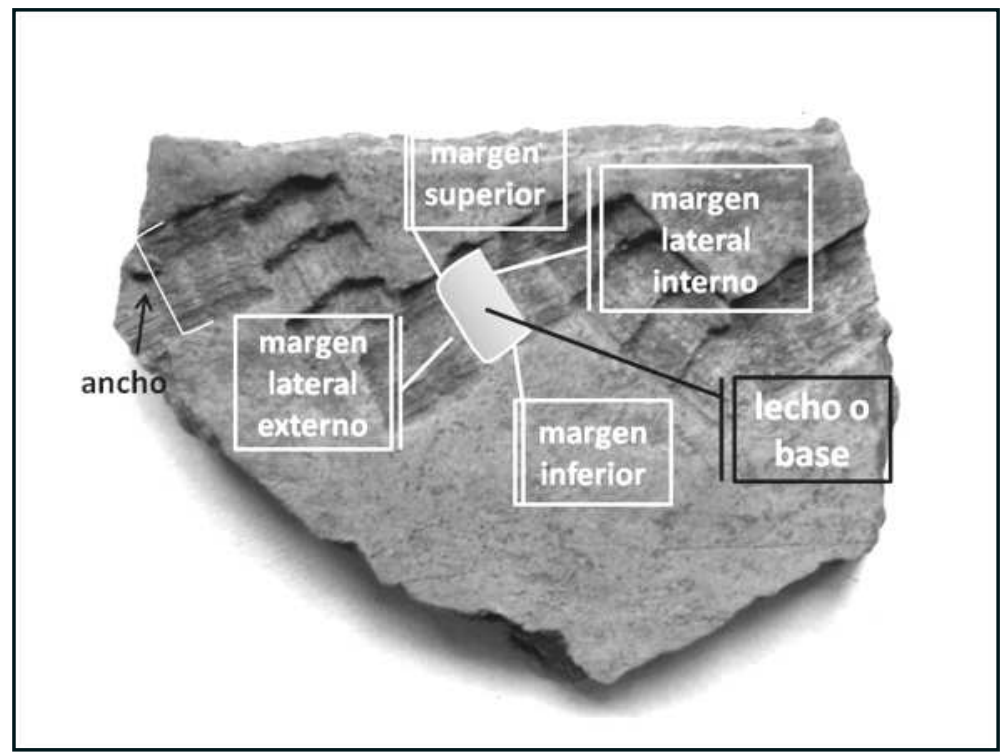

Figura 2. Segmentación artificial de una impronta efectuada mediante técnica de surco rítmico (tomado de Ottalagano 2010). 
experimentó realizando marcas inclinando el instrumento en un ángulo menor o mayor a $90^{\circ}$, o bien posicionándolo en ángulo recto con respecto a la línea formada por el trazo decorativo. Estas variaciones permitieron dar cuenta que, tanto la punta del elemento utilizado, como la manera en que el alfarero lo emplea durante la decoración, influyen en la morfología de las improntas ${ }^{2}$. Las piezas se dejaron secar, y fueron cocidas bajo la modalidad de cocción abierta (Ottalagano 2010). A fin de lograr una observación detallada, tanto de las improntas experimentales como de las arqueológicas, se las segmentó artificialmente; de manera tal de poder considerar las características de los diferentes márgenes y zonas que se forman cuando el instrumento es aplicado sobre la superficie arcillosa (figura 2).

\section{Resultados}

La decoración por medio de incisión de surco rítmico y de punto, suele disponerse como guardas geométricas (e.g. líneas paralelas, zig-zag, escalonados) que se ubican en las proximidades de los bordes de las vasijas, o bien como detalles accesorios en asas modeladas zoomorfas y antropomorfas. Predominan las improntas incisas cuadrangulares y rectangulares (tabla 1), las cuales por lo general presentan simetría entre márgenes superiores e inferiores. De acuerdo a las observaciones experimentales, los incisivos de coipo, los labios de recipientes fragmentados y las plumas, constituyen elementos cuya parte activa permite dejar improntas uniformes, claras y "limpias", comparables a las detectadas arqueológicamente en la muestra. En función de las semejanzas que evidencian las improntas arqueológicas con aquellas realizadas experimentalmente con estos elementos, se hace posible pensar que los mismos hayan podido ser utilizados en los distintos sectores fluviales considerados (figuras 3,4 y 5).

No obstante, se identifica un registro diferencial de improntas triangulares, las cuales aparecen frecuentemente en la decoración de la cerámica del Uruguay inferior (tabla 1). Este registro diferencial podría indicar, tanto una manera específica de utilizar un instrumental dado, o quizás más bien el uso de un instrumental particular en el sector inferior del río Uruguay. Algunas de las huellas triangulares registradas arqueológicamente, guardan similitud con aquellas realizadas experimentalmente con puntas óseas (figura 5, punto 3 y 4 ).

\begin{tabular}{|c|c|c|c|c|c|c|c|c|c|c|c|c|c|c|c|}
\hline \multicolumn{2}{|c|}{$\begin{array}{l}\text { Atributos de las } \\
\text { improntas }\end{array}$} & \multicolumn{2}{|c|}{$\begin{array}{c}\text { Paraná } \\
\text { Medio } \\
(n=1419)\end{array}$} & \multicolumn{2}{|c|}{$\begin{array}{l}\text { Paraná } \\
\text { Inferior } \\
(n=10)\end{array}$} & \multicolumn{2}{|c|}{$\begin{array}{l}\text { Uruguay } \\
\text { Inferior } \\
(n=62)\end{array}$} & \multicolumn{2}{|c|}{ Gestos técnicos } & \multicolumn{2}{|c|}{$\begin{array}{c}\text { Paraná } \\
\text { Medio } \\
(n=1419)\end{array}$} & \multicolumn{2}{|c|}{$\begin{array}{l}\text { Paraná } \\
\text { Inferior } \\
(n=10)\end{array}$} & \multicolumn{2}{|c|}{$\begin{array}{l}\text { Uruguay } \\
\text { Inferior } \\
(n=62)\end{array}$} \\
\hline \multirow{6}{*}{ 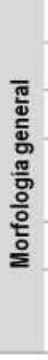 } & rectangular & 435 & $30,5 \%$ & 1 & $10 \%$ & 17 & $28 \%$ & \multirow{4}{*}{ 들 음 } & \multirow{3}{*}{$\begin{array}{c}\begin{array}{c}\text { profunda } \\
\text { (más de } 1 \mathrm{~mm})\end{array} \\
\begin{array}{c}\text { Intermedia } \\
(0,5-1 \mathrm{~mm})\end{array}\end{array}$} & \multirow[t]{2}{*}{84} & \multirow[t]{2}{*}{$6 \%$} & \multirow[t]{2}{*}{1} & \multirow[t]{2}{*}{$10 \%$} & \multirow[t]{2}{*}{9} & \multirow[t]{2}{*}{$14 \%$} \\
\hline & cuadrangular & 904 & $64 \%$ & \multirow{2}{*}{5} & $50 \%$ & 20 & $32 \%$ & & & & & & & & \\
\hline & triangular & 6 & $0,5 \%$ & & $0 \%$ & 7 & $11 \%$ & & & 1279 & $90 \%$ & 8 & $80 \%$ & 42 & $68 \%$ \\
\hline & $\begin{array}{l}\text { circular/oval/ } \\
\text { semicircular }\end{array}$ & 51 & $3.5 \%$ & 1 & $10 \%$ & 12 & $19 \%$ & & $\begin{array}{c}\text { poco prof. } \\
\text { (menor a } 0,5 \mathrm{~mm} \text { ) }\end{array}$ & 56 & $4 \%$ & 1 & $10 \%$ & 11 & $18 \%$ \\
\hline & trapezoidal & 0 & $0 \%$ & 0 & $0 \%$ & 2 & $3 \%$ & \multirow{2}{*}{ 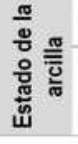 } & plástica & 29 & $2 \%$ & 2 & $20 \%$ & 2 & $3 \%$ \\
\hline & $\begin{array}{c}\text { otra/ } \\
\text { indefinida }\end{array}$ & 23 & $1,5 \%$ & 3 & $30 \%$ & 4 & $7 \%$ & & $\begin{array}{l}\text { dureza de } \\
\text { cuero }\end{array}$ & 1390 & $98 \%$ & 8 & $80 \%$ & 60 & $97 \%$ \\
\hline \multirow{3}{*}{$\begin{array}{l}\frac{5}{\omega} \\
\frac{0}{ \pm} \\
\text { Е }\end{array}$} & $1-3 \mathrm{~mm}$ & 928 & $65 \%$ & 7 & $70 \%$ & 35 & $57 \%$ & \multirow{3}{*}{ 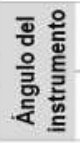 } & \multirow[t]{2}{*}{$<90^{\circ} 0>90^{\circ}$} & \multirow[t]{2}{*}{52} & $4 \%$ & 2 & $20 \%$ & 11 & $18 \%$ \\
\hline & $4-5 \mathrm{~mm}$ & 448 & $32 \%$ & 2 & $20 \%$ & 17 & $27 \%$ & & & & & & & & \\
\hline & más de $5 \mathrm{~mm}$ & 43 & $3 \%$ & 1 & $10 \%$ & 10 & $16 \%$ & & a $90^{\circ}$ & 1367 & $96 \%$ & 8 & $80 \%$ & 49 & $82 \%$ \\
\hline
\end{tabular}

Tabla 1. Atributos de las improntas incisas y gestos técnicos implementados. 


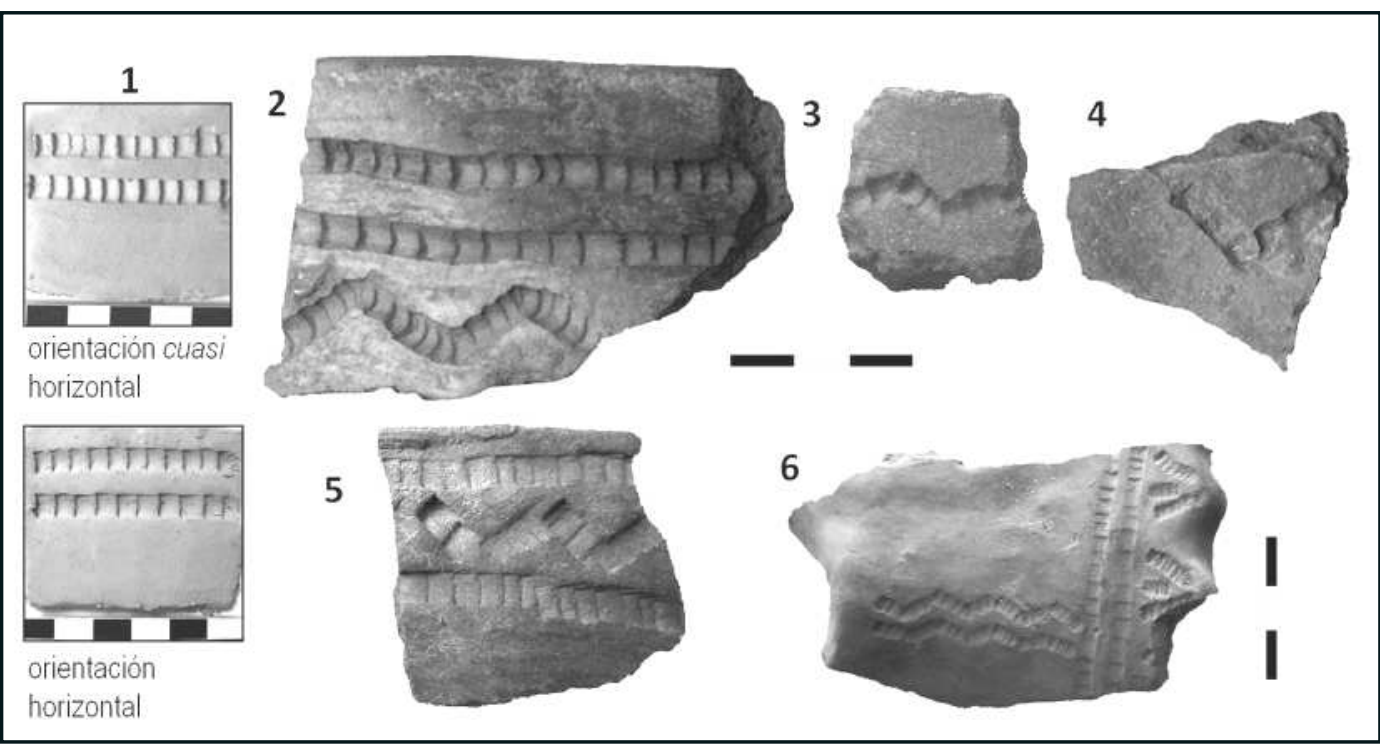

Figura 3. Comparación entre improntas arqueológicas y experimentales efectuadas con dientes de coipo. 1) huellas experimentales, según distintas orientaciones del instrumento; tiestos con surco rítmico: 2) y 6) Paraná medio, 3) y 5) Uruguay inferior, 4) Delta superior del Paraná.

Sin embargo, no se descarta que hayan podido utilizarse también artefactos líticos, materia prima predominante en la cuenca de este río.

Otro tipo de improntas arqueológicas, menos representadas en la muestra, se caracterizan por una morfología mayormente asimétrica, que concuerda generalmente con una irregula-

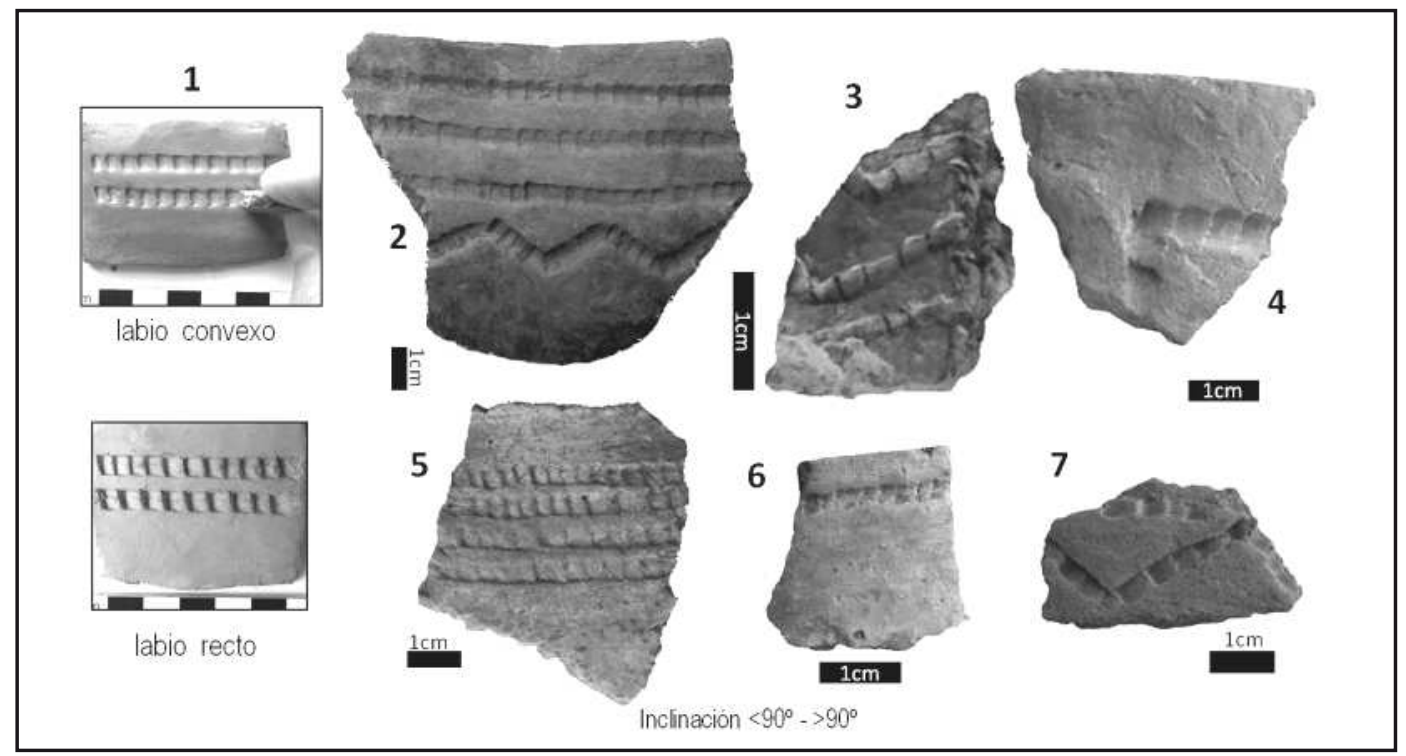

Figura 4. Comparación entre improntas arqueológicas y experimentales efectuadas con fragmentos de bordes cerámicos. 1) experimentación con labios convexos (posicionado a $90^{\circ}$ ) y rectos (inclinados a más de $90^{\circ}$ ); tiestos con surco rítmico: 2) y 5) Paraná medio, 3) y 6) Delta superior del Paraná, 4) y 7) Uruguay inferior. 


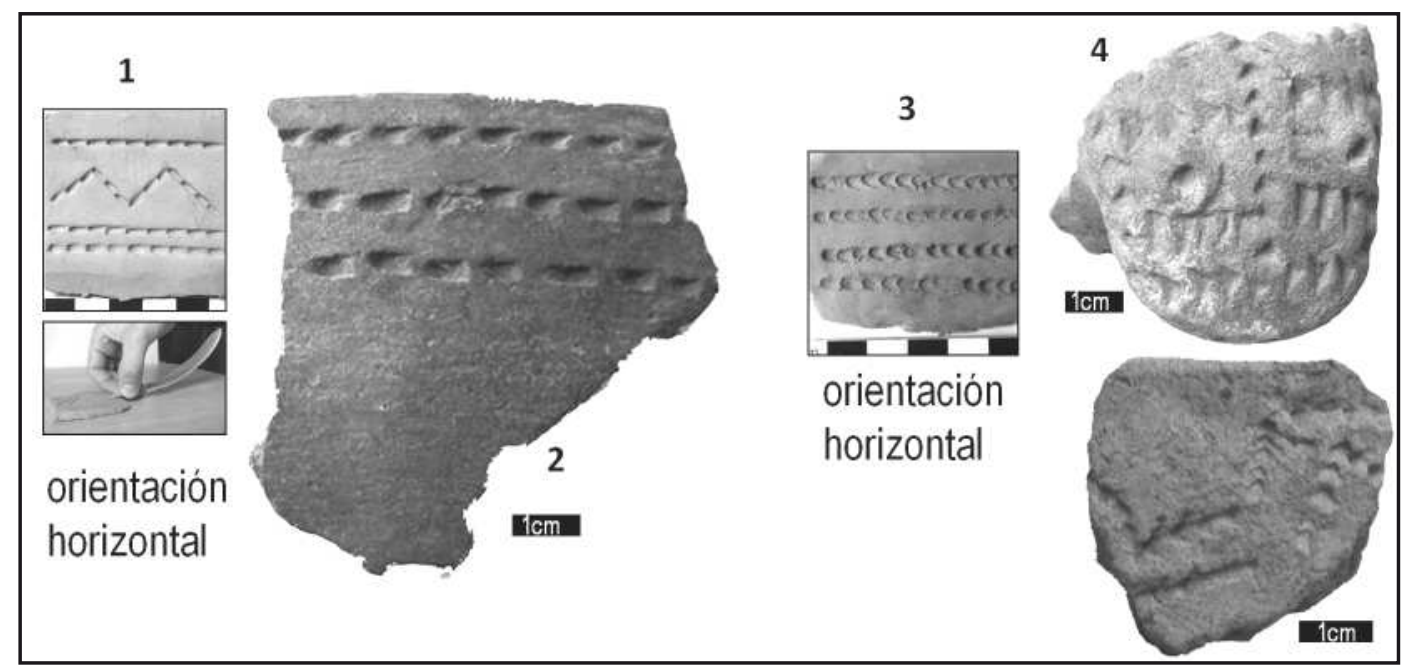

Figura 5. Comparación de improntas arqueológicas y experimentales. 1) experimentación con pluma; 2) incisión de punto del Uruguay inferior; 3) experimentación con punta ósea; 4) improntas arqueológicas triangulares del Uruguay inferior (incisión de surco rítmico y de punto).

ridad en la base de la impronta (figura 6). Las mismas se corresponden con la categoría de forma indefinida, y en menor medida, con la de circular-oval y rectangular ${ }^{3}$ planteada en la tabla 1. En este caso, la experimentación sugiere el uso de elementos con una superficie activa irregular, como pueden ser por ejemplo los huesos largos fracturados (e.g. Myocastor coypus) y, fundamentalmente, los restos de madera y vegetal (e.g. tallos de paja brava), los cuales tienden a generar improntas con abundantes microresiduos de pasta en la base de las mismas. En la figura 6 se señalan algunas semejanzas que pueden encontrarse entre las huellas experimentales efectuadas con estos elementos y aquellas arqueológicas, procedentes de las distintas zonas consideradas.

Por otro lado, siguiendo a Rye (1981) los análisis sobre los tiestos arqueológicos sugieren que la decoración se realizó cuando la arcilla se encontraba en estado de dureza de cuero. La profundidad de las improntas indica que éstas fueron efectuadas con una presión intermedia por parte del alfarero. Por último, el ángulo de inclinación con que se posicionó el instrumento, según se infiere de los datos experimentales (Ottalagano 2010), debió estar situado ampliamente a $90^{\circ}$. Es decir, que la parte activa del instrumento utilizado y la línea generada por el trazo decorativo, formaron un ángulo recto (tabla 1, figura 4).

\section{Conclusiones}

Las improntas arqueológicas estudiadas pueden ser divididas en: 1) las que presentan márgenes simétricos y morfología clara, y 2) las que poseen márgenes superiores e inferiores mayormente asimétricos, y no cuentan por lo general con formas nítidas. Por otra parte, se pueden diferenciar aquellas: 3) sin irregularidades en la base de la impronta, y 4) con irregularidades en la base de la impronta. Por lo común, las características 1 y 3 se dan simultáneamente. En el caso analizado, este grupo se corresponde mayormente con huellas de forma cuadrangular y rectangular, y son las más frecuentes en la muestra cerámica de los tres sectores fluviales abordados. Considerando los datos experimentales, sugieren la 


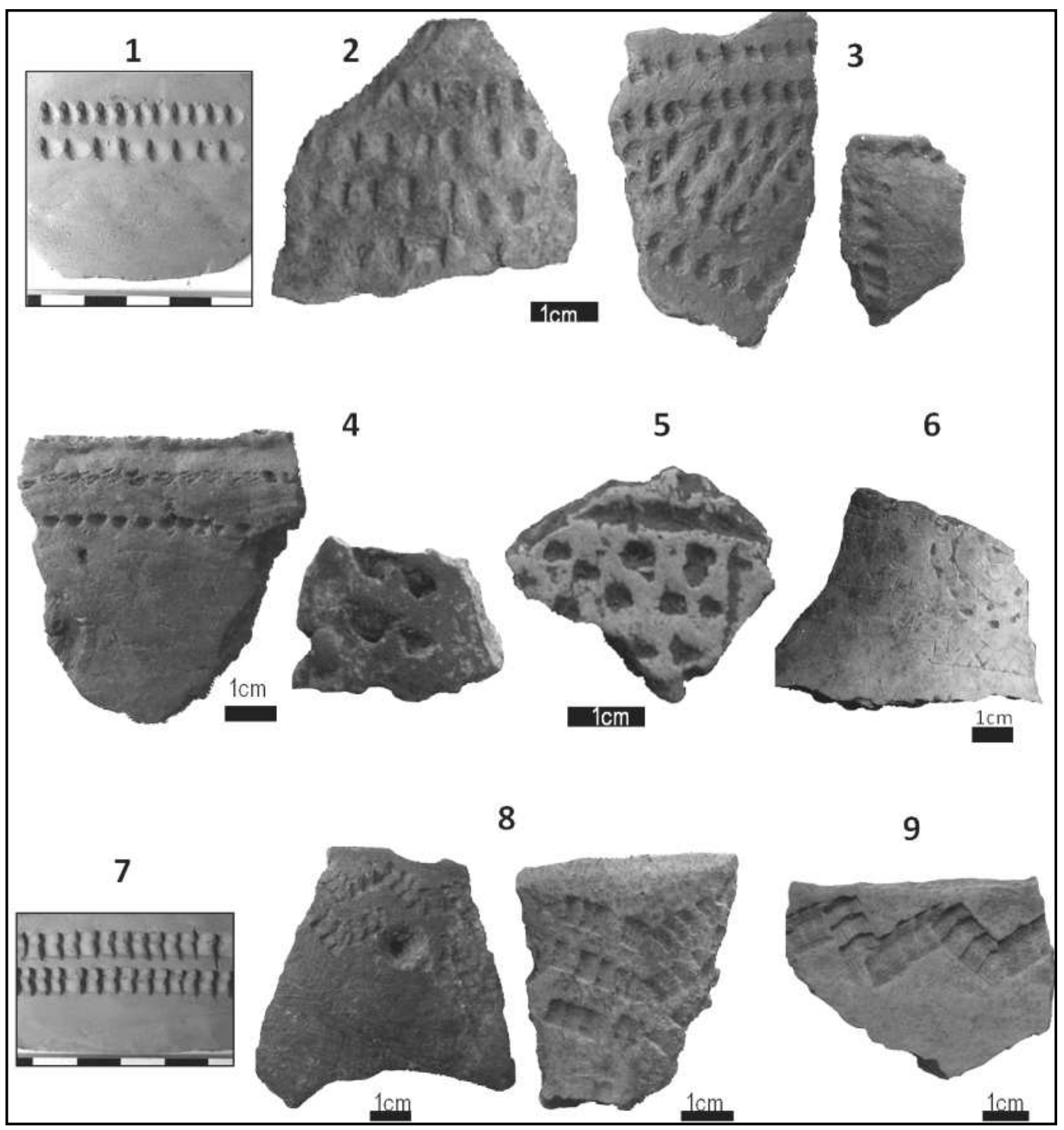

Figura 6. Comparación entre improntas arqueológicas y experimentales. 1) experimentación con tallos de paja brava (orientación cuasi perpendicular); improntas arqueológicas (incisión de punto): 2) Paraná medio, 3) Uruguay inferior; improntas arqueológicas indefinidas (incisión de punto): 4) Uruguay inferior, 5) Paraná medio, 6) Delta superior del Paraná; 7) experimentación con huesos largos fracturados; improntas arqueológicas (surco rítmico): 8) Uruguay inferior, 9) Paraná medio.

utilización de instrumentos tales como dientes de coipo, plumas y labios de recipientes fragmentados. De manera semejante, las características 2 y 4 suelen confluir. Este grupo de improntas, en tanto, se encuentra menos representado en la muestra; y presenta semejanza con las huellas experimentales realizadas con elementos tales como fragmentos óseos, madera y vegetal. 
En este sentido, se destacan similitudes importantes en la elaboración de la decoración incisa procedente de sitios arqueológicos localizados sobre las costas del los ríos Paraná y Uruguay, en los tramos considerados. Sin embargo cabe mencionar algunas diferencias que sugieren patrones específicos en la manufactura de lo decorativo en algunos sectores. Entre éstos puede señalarse el registro diferencial de improntas triangulares, proporcionalmente más frecuentes en los materiales arqueológicos del río Uruguay; lo cual posiblemente sugiere el uso de algún instrumental particular en esta zona, como por ejemplo artefactos óseos.

Estas conclusiones preliminares, abren el camino para considerar la necesidad de incrementar los estudios experimentales, así como de ampliar progresivamente la muestra bajo estudio, a medida que avancen los trabajos de campo en los sitios abordados. Esto se presenta como un requisito a futuro, que permitirá fortalecer o confrontar los resultados aquí publicados. En este sentido, este trabajo constituye un primer paso hacia un abordaje que tenga en cuenta, de manera comparativa, los aspectos tecnológicos de la decoración cerámica, dando la posibilidad de examinar los comportamientos de estas variables a escala regional.

\section{Notas}

${ }^{1}$ Se cuenta con tres fechados para los sitios del Paraná medio: $900( \pm 120)$ AP y $1380( \pm 100)$ AP, sitio ELI; y 950 ( \pm 120 ) años AP, sitio LM1; realizados sobre muestras de carbón, INGEIS (Ceruti 2003). Para el río Uruguay se dispone de un fechado sobre carbón de 1060 ( \pm 60$)$ años AP (LP-2590) en el sitio CDB3 (Castro 2011).

${ }^{2} \mathrm{El}$ grado en que uno $\mathrm{u}$ otro factor ejerce influencia sobre la forma de la impronta, depende en muchos casos del elemento utilizado. Así por ejemplo, las plumas pueden llegar a generar distintas clases de marcas, variando la manera en que se utiliza este elemento.

${ }^{3}$ En estos casos se tratarían de formas sub-rectangulares o sub-circulares.

\section{Bibliografía citada}

Badano, V.

1957 El arte plástico de los ribereños paranaenses. Memorias del Museo de Entre Ríos 34: $1-94$.

Castro, J. C.

2011 Arqueología en la cuenca del río Uruguay (Provincia de Entre Ríos). Cazadores Recolectores del Cono Sur 5. En prensa.

Ceruti, C.

2003 Entidades culturales presentes en la cuenca del Paraná Medio (margen entrerriana). Mundo de Antes 3: 111-135.

Ceruti, C. y M.B. Traver Borny

2007 Un aporte a la arqueología experimental: reproducción de material cerámico de la Entidad Goya-Malabrigo. Arqueología Argentina en los inicios de un nuevo siglo (ed. por F. Oliva, N. De Grandis y J. Rodríguez), pp.157-166. Laborde, Rosario. 
Cione, A. L.; Rizzo, A. y E. P. Tonni

1977 Relación cultura indígena-medio ambiente en un sitio de Rincón de Landa, Gualeguaychú, Entre Ríos, Republica Argentina. Nota preliminar. V Encuentro de Arqueología del Litoral. Fray Bentos, Uruguay.

Convención Nacional de Antropología

1966 Primera Convención Nacional de Antropología. UNC, Córdoba.

Frére, M.; González, M.I. y A. Francese

2004 Experimentación y diseño decorativo: primeros ensayos. La Región Pampeana: su Pasado Arqueológico (ed. por C. Gradin y F. Oliva), pp. 115-121. Laborde, Bs. As.

González, M. I.; Frére, M. y D. Fiore

2007 Redes de Interacción en el curso inferior y medio del Salado. Arqueología en las Pampas (ed. por C. Bayón, A. Pupio, M. I. González, N. Flegenheimer y M. Frére), pp. 365-384. Sociedad Argentina de Antropología, Bs. As.

Ottalagano, F.

2010 Decoración experimental de cerámica aplicada al estudio de las técnicas incisas del área del Paraná. Intersecciones en Antropología 11: 237-247.

Ottalagano, F.; Darigo, M.; Sulich, K. y L. Arelovich

2010 Investigaciones arqueológicas en el Delta superior entrerriano: sitio Cerro Puesto Acosta (Dpto. Victoria, Entre Ríos). Arqueología Argentina en el Bicentenario de la Revolución de Mayo (ed. por R. Bárcena y H. Chiavazza), pp. 1475-1480. UNCuyo, Mendoza.

Rye, O.

1981. Pottery technology. Taraxacum, Washington.

Serrano, A.

1946 Arqueología del Arroyo Las Mulas en el noroeste de Entre Ríos. Universidad de Córdoba, Córdoba. 